\title{
Weight and skin colour as predictors of vitamin $D$ status: results of an epidemiological investigation using nationally representative data
}

\author{
Sonali Rajan ${ }^{1, *}$, Tom Weishaar ${ }^{1}$ and Bryan Keller ${ }^{2}$ \\ 'Department of Health and Behavior Studies, Teachers College, Columbia University, 525 West 120th Street, \\ New York, NY 10027, USA: ${ }^{2}$ Department of Human Development, Teachers College, Columbia University, New \\ York, NY, USA
}

Submitted 25 June 2015: Final revision received 5 January 2016: Accepted 20 January 2016: First published online 12 February 2016

\begin{abstract}
Objective: Current US dietary recommendations for vitamin D vary by age. Recent research suggests that body weight and skin colour are also major determinants of vitamin D status. The objective of the present epidemiological investigation was to clarify the role of age as a predictor of vitamin D status, while accounting for body weight and skin colour, among a nationally representative sample.

Design: We calculated the mean serum 25-hydroxyvitamin D levels for the US population by age and weight, as well as by weight and race/ethnicity group. Multiple regression analyses were utilized to evaluate age and weight as predictors of vitamin D status: serum 25-hydroxyvitamin D levels with age alone, age and body weight, and age, body weight and their two-way interaction were modelled for the entire sample and each age subgroup. Graphical data were developed using B-spline non-linear regression.

Setting: National Health and Nutrition Examination Survey (31934 unweighted cases).

Subjects: Individuals aged 1 year and older.

Results: There were highly significant differences in mean vitamin D status among US residents by weight and skin colour, with those having darker skin colour or higher body weight having worse vitamin D status. Although a significant factor, the impact of age on vitamin D status was notably less than the impact of body weight. Conclusions: Vitamin D status varied predominantly by body weight and skin colour. Recommendations by nutritionists for diet and supplementation needs should take this into account if vitamin D-related health disparities are to be meaningfully reduced across the USA.
\end{abstract}

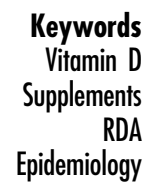

Keywords

Vitamin D

RDA

Epidemiology
The crucial role of vitamin D in contributing to one's overall quality of health is well established, yet African-Americans and Mexican-Americans continue to experience disparities in vitamin D status ${ }^{(1)}$. In addition to the evolving correlation between low vitamin D status and increased obesity risk, vitamin D deficiency is associated with many additional poor health outcomes, including low bone mineral density $^{(2)}$, poor skeletal health ${ }^{(3)}$, poor cardiovascular health $^{(4)}$, functional disabilities ${ }^{(5)}$, multiple sclerosis ${ }^{(6)}$ and poor immune system functioning ${ }^{(7)}$. In 2010, the Institute of Medicine (IOM) reported that vitamin D also has possible roles in carcinogenesis, diabetes, neuropsychological functions and pre-eclampsia ${ }^{(8)}$. Research has further confirmed that vitamin D deficiency has implications for children. Deficiency can impact their quality of health with regard to many health issues, including $\mathrm{CVD}^{(9)}$, insulin resistance $^{(10)}$ and anaemia ${ }^{(11)}$.
Currently, vitamin D daily requirements and supplementation recommendations by the IOM's Committee to Review Dietary Reference Intakes for Vitamin D and Calcium are driven primarily by age, with the recommendation of $10 \mu \mathrm{g} / \mathrm{d}$ ( $400 \mathrm{IU} / \mathrm{d})$ for infants increasing to $15 \mu \mathrm{g} / \mathrm{d}(600 \mathrm{IU} / \mathrm{d})$ after the first 12 months and increasing again to $53.3 \mu \mathrm{g} / \mathrm{d}(800 \mathrm{IU} / \mathrm{d})$ at age 70 years $^{(8)}$. The IOM supports the increase in vitamin $\mathrm{D}$ intake at age 70 years because of the variability in physiological changes that occur with ageing ${ }^{(8)}$. Further, the IOM cites several pieces of work establishing that vitamin D supplementation at $53.3 \mu \mathrm{g} / \mathrm{d}(800 \mathrm{IU} / \mathrm{d})$ in conjunction with sufficient $\mathrm{Ca}$ intake can reduce bone fracture risk among individuals over 70 years of age ${ }^{(8,12,13)}$. Work published over 20 years ago supports the notion that vitamin D status declines with age $^{(14,15)}$. To our knowledge, however, the present study is the first to use the most recent US National Health and 
Nutrition Examination Survey (NHANES) data to clarify the role of age as a predictor of vitamin D status, while accounting for other key factors, among a sample of youth and adults.

Indeed, recent research has established the importance of body weight and skin colour among both adults and children in establishing vitamin D recommendations ${ }^{(16,17)}$. For example, in 2014 work by Dhaliwal and colleagues identified the dose-response of vitamin D in obese individuals and concluded that those who weigh more may require up to $40 \%$ higher levels of vitamin D intake in comparison to their non-obese counterparts ${ }^{(18)}$. Work by $\mathrm{Ng}$ and colleagues also in 2014 determined the dose-response relationship between vitamin $\mathrm{D}$ and 25-hydroxyvitamin D (25(OH)D) concentrations in AfricanAmerican individuals and found that greater vitamin D intake was required to obtain required concentration levels among African-Americans ${ }^{(19)}$; thus providing additional evidence that darker-skinned individuals have greater vitamin D supplementation needs. Yet another recent publication computed the required supplemental vitamin D dose, by weight and skin colour, to help ensure children and adolescents meet the IOM's recommendations ${ }^{(17)}$. Despite these collective findings, the role of age when accounting for body weight and skin colour remains unclear with regard to recommending vitamin $\mathrm{D}$ requirements.

The purpose of the current work is to establish the role of age as a predictor of vitamin $\mathrm{D}$ status, while also accounting for body weight and race/ethnicity as a proxy for skin colour, among a nationally representative sample. We also discuss the implications of these findings for nutritionists and public health professionals.

\section{Methods and materials}

\section{Data source and management}

We used a subset of the data available from the continuous version of the NHANES ${ }^{(20)}$. NHANES researchers collect data on a two-year cycle at randomly selected US sites. Serum 25(OH)D data for individuals aged 1 year and older are currently available in four cycles of NHANES (2003-2004, 2005-2006, 2007-2008, 2009-2010). Other NHANES variables included in our analysis were self-reported race/ethnicity, self-reported age in months converted to decimal years, and measured body weight in kilograms. NHANES includes five race/ethnicity categories. In estimates for the entire US population we included all five. However, in the analysis by race/ ethnicity we did not use two of these ('Other Hispanic' and 'Other') because the groups do not have sufficient data to analyse separately. For that analysis we therefore retained three NHANES race/ethnicity categories: non-Hispanic black, Mexican-American and non-Hispanic white, each of which has enough data for nationally representative estimates.
For the population-level analyses there are 31934 (unweighted) cases. Of these, 12817 are individuals aged 19 years or less and 19117 are aged 20 years or more. For the analysis by race/ethnicity, after dropping cases in the race/ethnicity categories we did not use, there remained 28105 (unweighted) cases: 7261 non-Hispanic blacks, 7578 Mexican-Americans and 13266 non-Hispanic whites.

\section{Statistical analyses}

NHANES uses a complex survey design, which is presented in extensive detail elsewhere ${ }^{(21)}$. We used the statistical program R, version $3 \cdot 1 \cdot 1^{(22)}$ and its associated Survey package, version $3 \cdot 28-2^{(23)}$, which are appropriate for analysing complex survey data ${ }^{(24)}$. All analyses used NHANES-provided sample weights that adjust for unequal probabilities of selection (some sub-populations were oversampled), nonresponse adjustments and other adjustments ${ }^{(25)}$. Because the serum $25(\mathrm{OH}) \mathrm{D}$ measures were made on blood samples collected in NHANES mobile examination units, we used the mobile examination unit weights.

Given the significant role age has historically played in serving as a basis for making current vitamin D supplementation recommendations ${ }^{(8)}$, we generated nine regression models in three age groups: (i) participants of all ages; (ii) participants aged 19 years and younger; and (iii) participants aged 20 years and older. For each age group we modelled serum 25(OH)D levels with age alone, with age and body weight, and with age, body weight and their two-way interaction. We developed the graphical data using B-spline non-linear regression with two knots calculated to split the cases in each analysis into three equal-sized (weighted) groups. Confidence intervals for this analysis, as shown in the graphs, vary across the range of ages and weights: they are wider where there are fewer cases and narrower where there are more cases.

\section{Results}

The demographic characteristics of the weighted NHANES data are of the same as the non-institutionalized US population $^{(21,25)}$ aged 1 year and above. A breakdown of the sample demographics is presented in Table 1 . Table 2 shows results of regression models in each of the three groups. In all groups, likelihood ratio tests and Akaike's information criteria favoured models including two-way interactions (models 3, 6 and 9) over the simpler models.

To more clearly evaluate the role of age and body weight in predicting vitamin D status, it is possible to simplify interpretation of the significant interaction between age and body weight by examining conditional regression equations. For example, for the group of participants aged 20 years and older, the baseline regression coefficients are the ones for model 9 (Table 2). Fixing age to a value of 20 years, the youngest age in that subset, 
Table 1 Demographics of the sample of participants from the National Health and Nutrition Examination Survey, 2003-2010

\begin{tabular}{|c|c|c|c|c|c|c|}
\hline & \multicolumn{2}{|c|}{ All participants ( $n$ 31934) } & \multicolumn{2}{|c|}{ Aged $1-<20$ years $(n 12817)$} & \multicolumn{2}{|c|}{ Aged $\geq 20$ years $(n 19117)$} \\
\hline & $\%$ or Mean & SE & $\%$ or Mean & SE & $\%$ or Mean & SE \\
\hline \multicolumn{7}{|l|}{ Sex } \\
\hline Male & $49 \cdot 0$ & 0.0 & 51.9 & 0.01 & $48 \cdot 1$ & 0.0 \\
\hline Female & $51 \cdot 0$ & 0.0 & 48.0 & 0.01 & 51.9 & 0.0 \\
\hline \multicolumn{7}{|l|}{ Race/ethnicity } \\
\hline Non-Hispanic white & $68 \cdot 2$ & 0.02 & 58.5 & 0.02 & $72 \cdot 2$ & 0.02 \\
\hline Non-Hispanic black & 11.6 & 0.01 & 14.5 & 0.01 & 10.7 & 0.01 \\
\hline Other & $6 \cdot 0$ & 0.0 & 6.7 & 0.01 & $5 \cdot 7$ & 0.0 \\
\hline Mexican American & $9 \cdot 6$ & 0.01 & $14 \cdot 4$ & 0.01 & 8.2 & 0.01 \\
\hline Other Hispanic & 4.6 & 0.01 & $5 \cdot 9$ & 0.01 & $4 \cdot 2$ & 0.01 \\
\hline Mean age (years) & 38.7 & 0.28 & $11 \cdot 2$ & 0.09 & $47 \cdot 2$ & 0.28 \\
\hline Mean weight $(\mathrm{kg})$ & $73 \cdot 3$ & 0.29 & $45 \cdot 9$ & 0.41 & 81.7 & 0.28 \\
\hline Mean serum $25(\mathrm{OH}) \mathrm{D}(\mathrm{ng} / \mathrm{ml})$ & $26 \cdot 3$ & 0.26 & $27 \cdot 4$ & 0.32 & $25 \cdot 9$ & 0.27 \\
\hline
\end{tabular}

25(OH)D, 25-hydroxyvitamin D.

Table 2 Summary of regression analysis results

\begin{tabular}{|c|c|c|c|c|c|c|}
\hline Model & Predictor(s) & Model coefficients & Model intercept & $R^{2}$ value & $\begin{array}{l}\text { Wald test } \\
\text { statistic }^{*}\end{array}$ & $\begin{array}{c}\text { Model } \\
\text { significance }\end{array}$ \\
\hline 1 & Age (all participants) & $\beta_{\text {age }}=-0.015 \dagger$ & $26 \cdot 8$ & 0.001 & $F=7 \cdot 2$ & $P=0.009$ \\
\hline 2 & Age (all participants) and body weight & $\begin{array}{l}\beta_{\text {age }}=0.031 \dagger \\
\beta_{\text {weight }}=-0.081 \dagger\end{array}$ & $31 \cdot 0$ & 0.042 & $F=362$ & $P<0.001$ \\
\hline 3 & $\begin{array}{l}\text { Age (all participants), body } \\
\text { weight and two-way interaction }\end{array}$ & $\begin{array}{l}\beta_{\text {age }}=0.009 \\
\beta_{\text {weight }}=-0.090 \dagger \\
\beta_{\text {age } x \text { weight }}=0.00003 \dagger\end{array}$ & 31.5 & 0.042 & $F=272 \cdot 3$ & $P<0.001$ \\
\hline 4 & Age (participants aged $1-<20$ years) & $\beta_{\text {age }}=-0.316 \dagger$ & $31 \cdot 0$ & 0.037 & $F=121 \cdot 6$ & $P<0.001$ \\
\hline 5 & $\begin{array}{l}\text { Age (participants aged } 1-<20 \text { years) } \\
\text { and body weight }\end{array}$ & $\begin{array}{l}\beta_{\text {age }}=0.096 \dagger \\
\beta_{\text {weight }}=-0.103 \dagger\end{array}$ & $31 \cdot 1$ & 0.063 & $F=207 \cdot 8$ & $P<0.001$ \\
\hline 6 & $\begin{array}{l}\text { Age (participants aged } 1-<20 \text { years), } \\
\text { body weight and two-way interaction }\end{array}$ & $\begin{array}{l}\beta_{\text {age }}=-0.043 \\
\beta_{\text {weight }}=-0.182 \dagger \\
\beta_{\text {age } \times \text { weight }}=0.00524 \dagger\end{array}$ & $33 \cdot 0$ & 0.066 & $F=167.9$ & $P<0.001$ \\
\hline 7 & Age (participants aged $\geq 20$ years) & $\beta_{\text {age }}=0.027 \dagger$ & $24 \cdot 6$ & 0.002 & $F=10.9$ & $P=0.002$ \\
\hline 8 & $\begin{array}{l}\text { Age (participants aged } \geq 20 \text { years) } \\
\text { and body weight }\end{array}$ & $\begin{array}{l}\beta_{\text {age }}=0.025 \dagger \\
\beta_{\text {weight }}=-0.080 \dagger\end{array}$ & $31 \cdot 2$ & 0.031 & $F=160 \cdot 3$ & $P<0.001$ \\
\hline 9 & $\begin{array}{l}\text { Age (participants aged } \geq 20 \text { years), } \\
\text { body weight and two-way interaction }\end{array}$ & $\begin{array}{l}\beta_{\text {age }}=-0.038 \\
\beta_{\text {weight }}=-0.117 \dagger \\
\beta_{\text {age } \times \text { weight }}=0.00081 \dagger\end{array}$ & $34 \cdot 1$ & 0.032 & $F=112 \cdot 1$ & $P<0.001$ \\
\hline
\end{tabular}

For models $1-3$, unweighted $n$ is 31934 ; for models $4-6$, unweighted $n$ is 12817 ; for models 7-9, unweighted $n$ is 19117 .

${ }^{*}$ Complex survey analysis relies on the Wald test as an omnibus test of each model.

†Model coefficient significant at $P<0.05$.

results in the following conditional prediction equation:

$$
E[\text { serum vitamin } \mathrm{D} \mid \text { age }]=33 \cdot 3-0 \cdot 10 \times \text { weight } .
$$

Similarly, fixing age to a value of 80 years results in the following conditional prediction equation:

$$
E[\text { serum vitamin } \mathrm{D} \mid \text { age }]=31 \cdot 1-0 \cdot 05 \times \text { weight } .
$$

By comparing the two models, we see clearly that the relationship between vitamin D status and body weight is moderated by age such that for the youngest in this subset (20-year-olds), each additional kilogram increase corresponds to an expected serum vitamin D loss of $0.10 \mathrm{ng} / \mathrm{ml}$, whereas for the eldest individuals (80-year-olds), each additional kilogram increase corresponds to an expected vitamin D loss of $0.05 \mathrm{ng} / \mathrm{ml}$. That is, although weight plays a significant role across all ages, it has a larger impact on vitamin D status for younger adults than it does for older adults. The same relationship applies in the other two groups: the expected loss of vitamin D status in $\mathrm{ng} / \mathrm{ml}$ decreases with age. Furthermore, we can track the main effect of age by comparing intercepts: a change of 60 years in age corresponds to an expected decrease in vitamin D status of $33 \cdot 3-31 \cdot 1=2 \cdot 26 \mathrm{ng} / \mathrm{ml}$.

To further understand the role of age, body weight and skin colour in predicting vitamin D status, we illustrate the relationships in Figs 1-4. Figure 1 shows the estimated mean serum $25(\mathrm{OH}) \mathrm{D}$ level of US residents, with $95 \% \mathrm{CI}$, over the range of ages available in NHANES (NHANES recodes individuals over 80 years old to 80 years to protect their privacy). Children have higher mean vitamin $\mathrm{D}$ levels than adults. Figure 2 shows mean serum 25(OH)D levels over the normal range of body weights. There is a clear clinically and statistically significant decline in vitamin D status as body weight increases. Figures 3 and 4 separate out the means and $95 \%$ CI for non-Hispanic whites, Mexican-Americans and non-Hispanic blacks by age and body weight, respectively. 


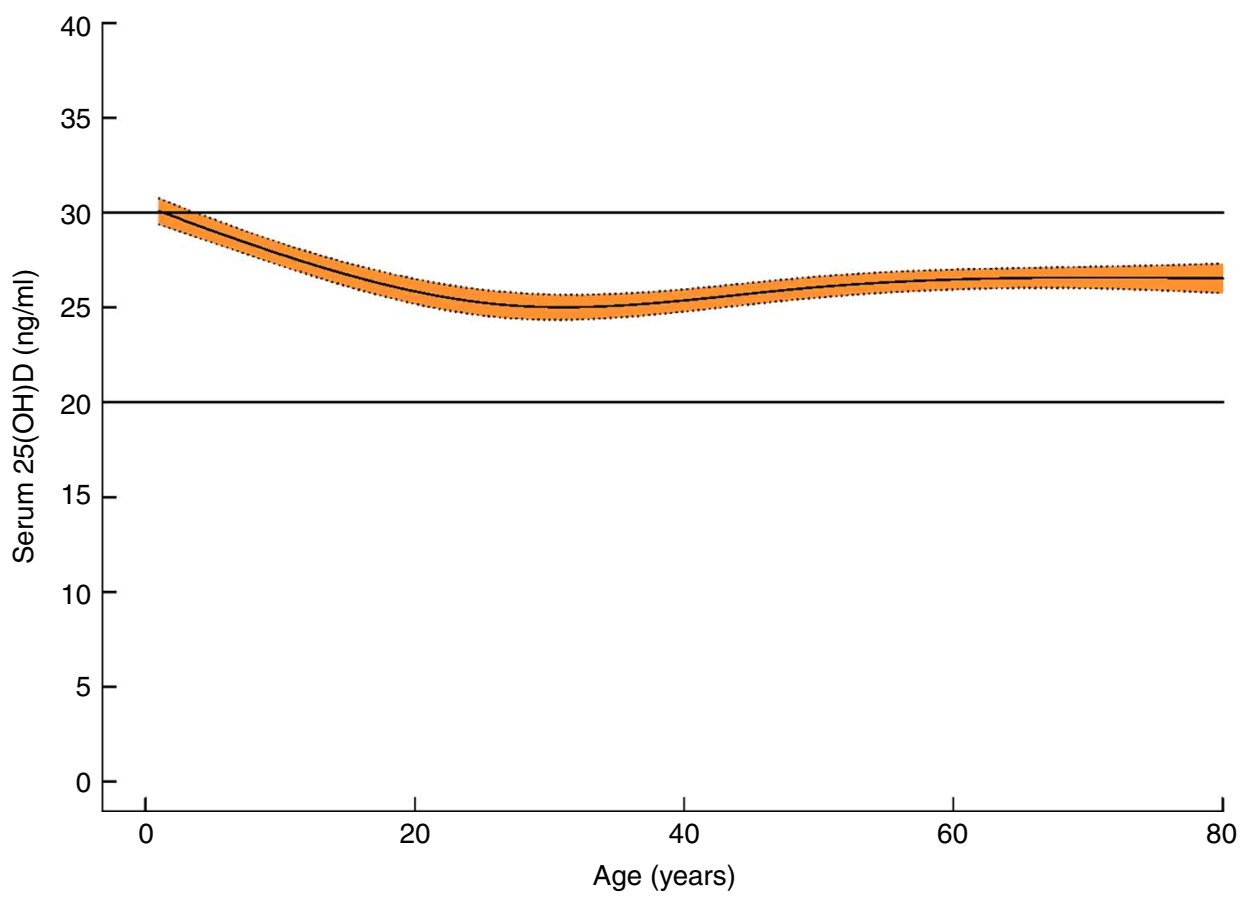

Fig. 1 Estimated mean serum 25-hydroxyvitamin D (25(OH)D) level, with $95 \%$ confidence interval, of $\geq 1$-year-old US residents over the range of ages available in the National Health and Nutrition Examination Survey, 2003-2010. These graphical data were developed using B-spline non-linear regression; the confidence interval varies across the range of ages. The line at $20 \mathrm{ng} / \mathrm{ml}$ and $30 \mathrm{ng} / \mathrm{ml}$ represents the population average recommended by the Institute of Medicine ${ }^{(8)}$ and the Endocrine Society ${ }^{(39)}$, respectively. Children have higher mean vitamin $D$ levels than adults

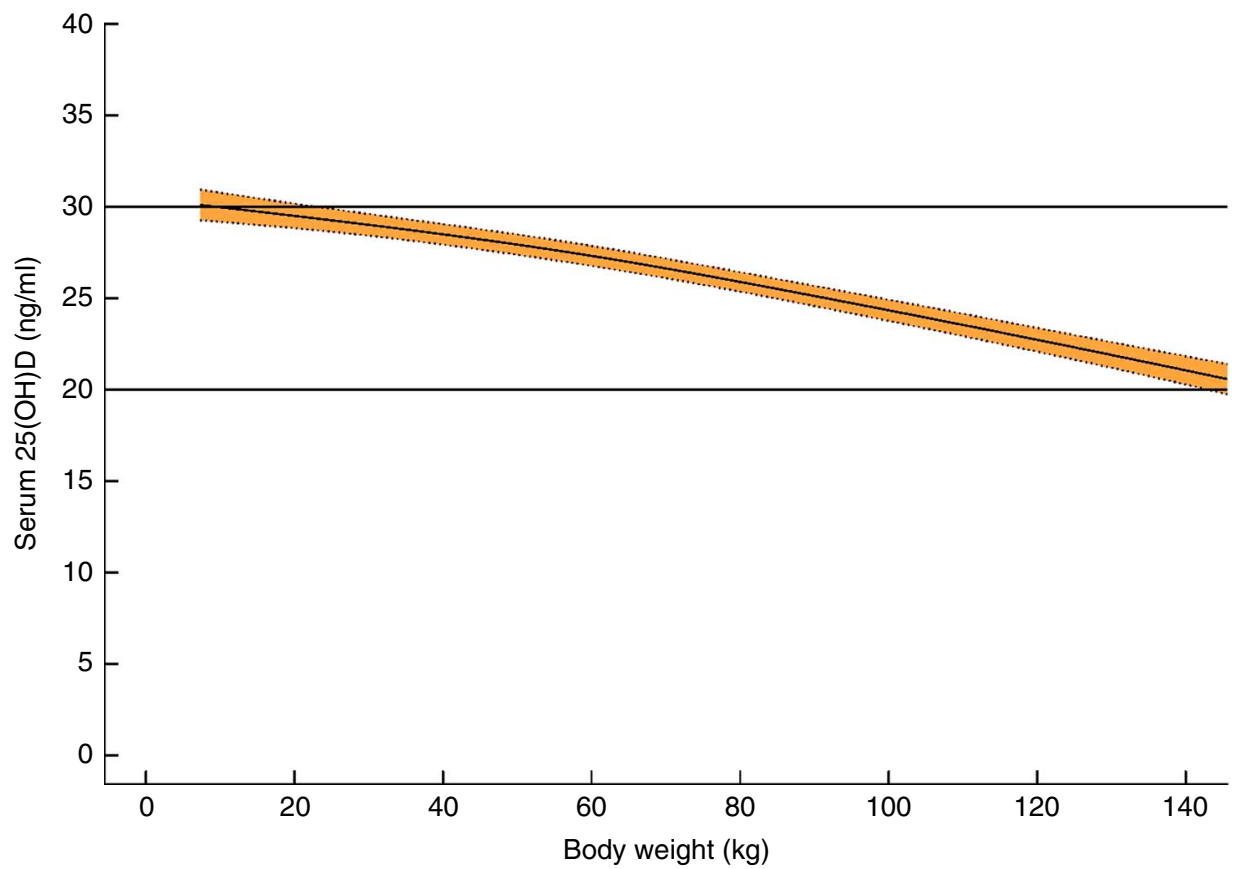

Fig. 2 Estimated mean serum 25-hydroxyvitamin D (25(OH)D) level, with $95 \%$ confidence interval, of $\geq 1$-year-old US residents over the normal range of body weights available in the National Health and Nutrition Examination Survey, 2003-2010. These graphical data were developed using B-spline non-linear regression; the confidence interval varies across the range of body weights. The line at $20 \mathrm{ng} / \mathrm{ml}$ and $30 \mathrm{ng} / \mathrm{ml}$ represents the population average recommended by the Institute of Medicine ${ }^{(8)}$ and the Endocrine Society ${ }^{(39)}$, respectively. There is a statistically significant decline in vitamin D status as body weight increases

Resulting from limited production of vitamin $\mathrm{D}$ in darker skin, non-Hispanic blacks, on average, have much lower levels of serum $25(\mathrm{OH}) \mathrm{D}$ than other groups. On average,
non-Hispanic blacks who are older than 16 years have serum $25(\mathrm{OH}) \mathrm{D}$ levels below $20 \mathrm{ng} / \mathrm{ml}$ with an increase noted among the eldest segment of the sample. Similarly, and 


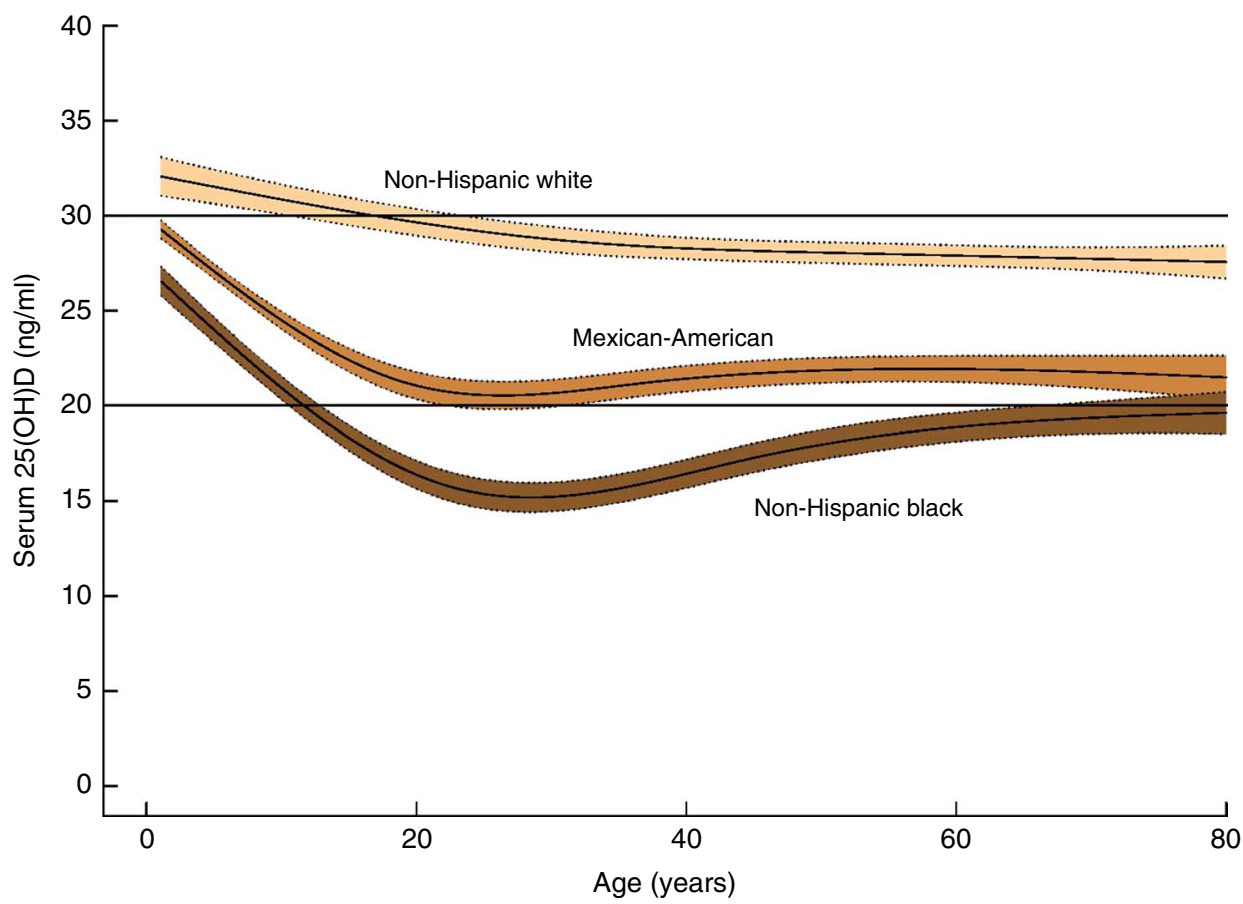

Fig. 3 Estimated mean serum 25-hydroxyvitamin D (25(OH)D) level, with $95 \%$ confidence interval, of $\geq 1$-year-old US residents, by skin colour, over the range of ages available in the National Health and Nutrition Examination Survey, 2003-2010. These graphical data were developed using B-spline non-linear regression; the confidence interval varies across the range of ages. The line at $20 \mathrm{ng} / \mathrm{ml}$ and $30 \mathrm{ng} / \mathrm{ml}$ represents the population average recommended by the Institute of Medicine ${ }^{(8)}$ and the Endocrine Society ${ }^{(39)}$, respectively. On average, non-Hispanic blacks older than 16 years have vitamin $D$ levels below $20 \mathrm{ng} / \mathrm{ml}$, with an increase noted among the eldest segment of the sample

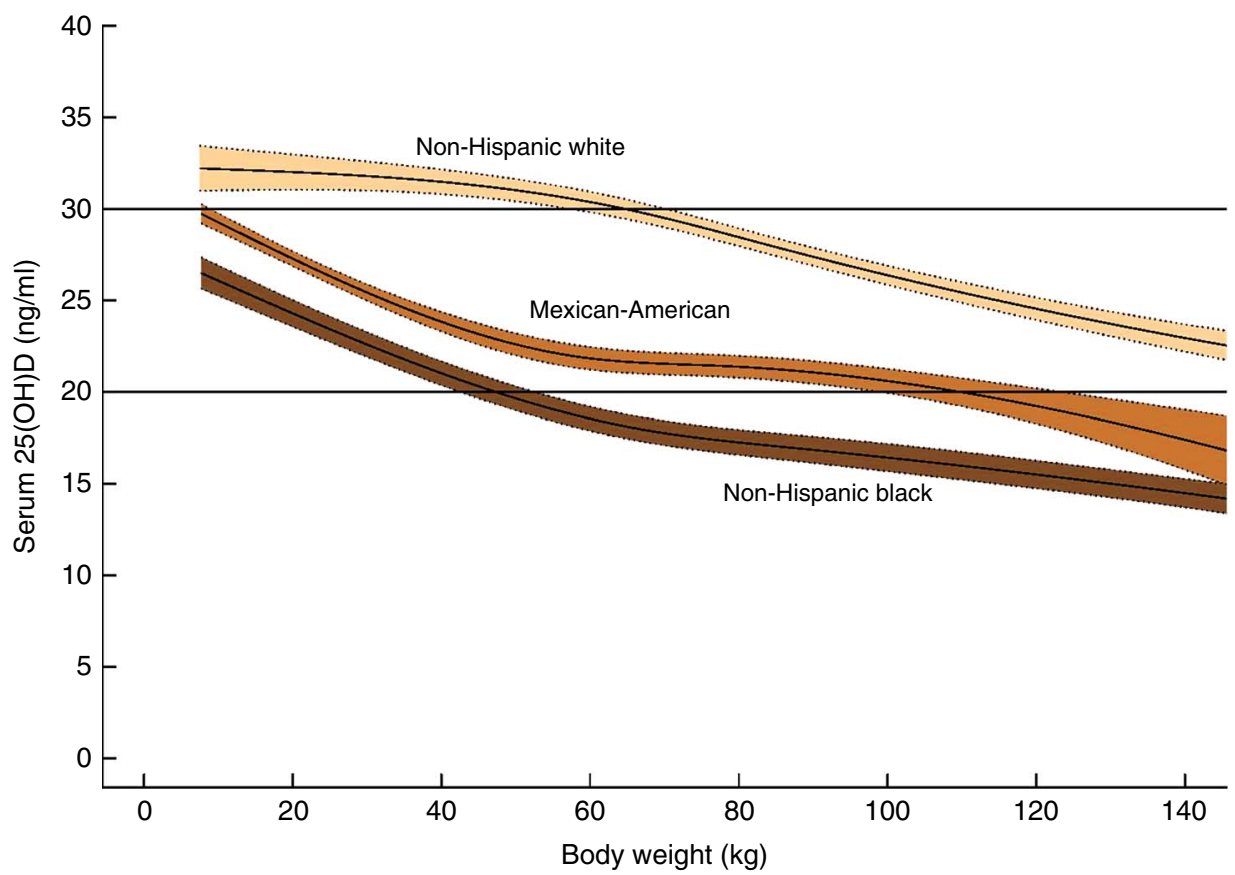

Fig. 4 Estimated mean serum 25-hydroxyvitamin D (25(OH)D) level, with $95 \%$ confidence interval, of $\geq 1$-year-old US residents, by skin colour, over the normal range of body weights available in the National Health and Nutrition Examination Survey, 2003-2010. These graphical data were developed using B-spline non-linear regression; the confidence interval varies across the range of body weights. The line at $20 \mathrm{ng} / \mathrm{ml}$ and $30 \mathrm{ng} / \mathrm{ml}$ represents the population average recommended by the Institute of Medicine ${ }^{(8)}$ and the Endocrine Society ${ }^{(39)}$, respectively. Among all three subgroups there is a clear and significant decline in vitamin D status as body weight increases 
among all three subgroups, we see in Fig. 4 a clear and significant decline in vitamin D status as body weight increases, with non-Hispanic blacks weighing more than $50 \mathrm{~kg}$ and Mexican-Americans weighing more than $110 \mathrm{~kg}$ having seum 25(OH)D levels below $20 \mathrm{ng} / \mathrm{ml}$. Vitamin D status for both non-Hispanic blacks and Mexican-Americans remains significantly lower than for white individuals. Indeed, only white individuals have mean serum 25(OH)D levels above $20 \mathrm{ng} / \mathrm{ml}$ at all weight levels.

\section{Discussion}

\section{Summary of key findings}

The present study used nationally representative data to establish that weight and skin colour are statistically and clinically significant predictors of vitamin D status. We demonstrated that among all participants, decline in serum $25(\mathrm{OH}) \mathrm{D}$ status was associated predominantly with an increase in weight. Although still a significant factor, the impact of age as a predictor of vitamin D status was notably smaller in comparison to the impact of body weight. We also saw that the relationship between body weight and vitamin D status was moderated by age. Among individuals younger than 20 years old, we observed that age remains a significant predictor of vitamin D status after accounting for weight and vitamin D status appears to increase, not decrease, with age. Among individuals aged 20 years and older, our findings demonstrated that while both age and weight are significant predictors of vitamin D status, the impact of weight is notably larger than the impact of age on vitamin D status. Lastly, we illustrated conclusively that there are highly significant differences in mean vitamin D status by weight and skin colour in the US population.

\section{Study limitations}

There are several limitations of the study that ought to be taken into consideration when interpreting these findings. The results presented herein apply only to the US population. Although other populations more globally have exhibited deficiencies in vitamin $\mathrm{D}^{(26-28)}$, these differences may be the result of differences in latitude, food fortification or other reasons not accounted for in the present study. Additionally, factors beyond weight and skin colour do contribute to the variability observed in vitamin D status. Indeed, it must be acknowledged that there are three important ways to improve vitamin D status: exposure to sunlight, diet and supplementation. However, as noted in previous research, addressing vitamin D deficiency through diet alone is very difficult for most individuals ${ }^{(29)}$. An earlier study using NHANES data illustrated that dietary intake of vitamin $\mathrm{D}$ is lowest among non-Hispanic black and Mexican-American individuals ${ }^{(30)}$. And more recent research has demonstrated that dietary intake of vitamin D is a significant predictor of vitamin D status, explaining a notable proportion of the variance observed in vitamin D status among certain segments of the population ${ }^{(31)}$. Historically, vitamin D intake data from primarily white participants have been used to inform supplementation recommendations ${ }^{(8)}$. The present study did not include dietary intake as a predictor in these analyses; therefore future work should also include intake estimates from participants across a range of body weights and skin colours to most accurately predict vitamin D status. Further, there have been and continue to be considerable public health efforts to minimize sun exposure among the US population because of the direct risk of skin cancer ${ }^{(32)}$. As such, the only remaining method of meaningfully preventing vitamin $\mathrm{D}$ deficiency is through dietary supplementation.

The present study also did not look at other determinants of vitamin D status. However, previous work by one of the study authors established that skin colour and weight are more important determinants of vitamin D status in comparison to many other factors such as sex, tobacco use and socio-economic status ${ }^{(1)}$. Previous work has additionally demonstrated that body weight is a comparable measure to $\mathrm{BMI}^{(33)}$; individuals are more likely to know their own weight than their BMI. As such, this literature base together provided clear evidence for pursuing the present set of analyses. With regard to other potential confounders, although some variation in vitamin D status related to climate exists, data on latitude were not available via NHANES and information on season, despite being available, was not randomized and thus deemed inappropriate for use in the present study. It should be noted that given the established seasonal variation in vitamin D status, NHANES purposefully collects data from individuals located in northern/higher-latitude locations in the USA in the summer and from individuals located in southern/lower-latitude locations in the winter, to minimize weather-related cancellations ${ }^{(21)}$. Combined with the fact that the proportion of non-Hispanic blacks and Mexican-Americans is higher in the southern USA, the NHANES summer/winter data collection protocol adds unaccounted variability to the vitamin $\mathrm{D}$ data by population group. Indeed, the present results do not reflect vitamin D levels during the darker months; a time of greatest dietary need ${ }^{(34)}$. However, overall, the data here are consistent with other studies. In addition, although we used the available data for race/ethnicity groups as a proxy for skin colour, we acknowledge that skin colour is a variable biological characteristic and that some patients within specific racial/ethnic groups may accordingly have variable vitamin $\mathrm{D}$ needs.

Lastly, it should be recognized that there is a lack of definitive evidence specifically via randomized controlled trials that vitamin D status is a predictor of positive non-skeletal effects. As such, the need for increased vitamin D supplementation among African-American individuals specifically has been deemed 'premature'(8). However, health policy panels in the USA have 
simultaneously encouraged African-American individuals, at particular risk for vitamin D-related health disparities, to reduce their already low vitamin D levels by avoiding sunlight to minimize a possible increase in likelihood of developing skin cancer $^{(8)}$. This encouragement exists despite the lack of randomized controlled trials investigating the relationship between sunlight and skin cancer. Thus, while the availability of randomized controlled trials is most ideal for serving as the basis for dietary, supplementation and behaviour recommendations, from a broader public health perspective the importance of vitamin $\mathrm{D}$ in the context of disease prevention should not be underestimated. Moreover, a recent randomized controlled trial evaluating treatment regimens for vitamin D deficiency specifically among minority adolescents demonstrated that body weight is a significant factor impacting vitamin D status, thus providing some support for the findings presented here ${ }^{(35)}$.

\section{Implications for nutritionists and public bealtb professionals}

The results of the present study demonstrate that body weight and skin colour are meaningful predictors of vitamin D status. While the optimal level of the biomarker for vitamin $\mathrm{D}, 25(\mathrm{OH}) \mathrm{D}$, remains controversial, it is generally unrecognized by nutrition professionals that key determinants of vitamin D status in the USA, in addition to age and vitamin D dietary intake, are skin colour and body weight. Importantly, our present work illustrates conclusively that differences by age have a smaller impact on vitamin D status in comparison to the impact of body weight and skin colour. Given these findings, the recognition of the importance of supplementation for improving vitamin D status and the acknowledgement that serum $25(\mathrm{OH}) \mathrm{D}$ is an accurate indicator of vitamin D status ${ }^{(36)}$, recommended daily requirements for vitamin D should take into account the significant role of weight and skin colour. The quality of the data used to establish the present findings contributes to the strength of the study. Indeed, for the US population, NHANES observations are typically the gold standard to which results of other studies are compared ${ }^{(37,38)}$. However, as current available data on vitamin D status remain limited, public health researchers should focus on the collection of comprehensive and nationally representative data needed to determine appropriate RDA for vitamin $\mathrm{D}$ by weight and skin colour, in addition to accounting for the role of age and dietary intake. Lastly, public health efforts to reduce vitamin D-related health disparities more broadly via the use of supplementation should recognize that disparities exist across the range of body weight and skin colour. Therefore, targeted public health campaigns should emphasize these findings and encourage individuals to speak accordingly with a registered dietitian about vitamin $\mathrm{D}$ supplementation needs. Ultimately such efforts will contribute to improving health outcomes among multiple segments of the US population.

\section{Acknowledgements}

Financial support: This research received no specific grant from any funding agency in the public, commercial or not-for-profit sectors. Conflict of interest: None. Authorship: S.R. conceptualized the study with T.W., conducted the background research for the study and worked closely with T.W. and B.K. on the writing of the manuscript and the interpretation of the results. T.W. conceptualized the study with S.R., contributed to the writing of the manuscript, analysed the data, interpreted the results and worked with B.K. to develop the figures. B.K. contributed to the writing of the manuscript and worked directly with T.W. On the analysis of these data and development of the figures. Ethics of human subject participation: The National Center for Health Statistics Ethics Review Board approved the National Health and Nutrition Examination Survey (NHANES) data collection protocols and the Institutional Review Board of Teachers College, Columbia University determined our work with NHANES to be exempt from review.

\section{References}

1. Weishaar T \& Vergili JM (2013) Vitamin D status is a biological determinant of health disparities. J Acad Nutr Diet 113, 643-651.

2. Reid IR, Bolland MJ \& Grey A (2014) Effects of vitamin D supplements on bone mineral density: a systematic review and meta-analysis. Lancet 383, 146-155.

3. Soliman A, De Sanctis V \& Yassin M (2013) Vitamin D status in thalassemia major: an update. Mediterr J Hematol Infect Dis 5, e2013057.

4. Pilz S, Gaksch M, Kienreich K et al. (2015) Effects of vitamin $\mathrm{D}$ on blood pressure and cardiovascular risk factors: a randomized controlled trial. Hypertension 65, 1195-1201.

5. Kojima G, Tamai A, Masaki K et al. (2013) Prevalence of vitamin D deficiency and association with functional status in newly admitted male veteran nursing home residents. J Am Geriatr Soc 61, 1953-1957.

6. Pakpoor J \& Ramagopalan S (2015) Evidence for an association between vitamin $\mathrm{D}$ and multiple sclerosis. Curr Top Behav Neurosci 26, 105-115.

7. Bentley J (2013) Vitamin D deficiency: identifying gaps in the evidence base. Nurs Stand 27, 35-41.

8. Ross AC, Taylor CL, Yaktine AL et al. (editors) (2011) Dietary Reference Intakes for Calcium and Vitamin D. Washington, DC: The National Academies Press.

9. Nwosu BU, Maranda L, Cullen K et al. (2013) Vitamin D status is associated with early markers of cardiovascular disease in prepubertal children. J Pediatr Endocrinol Metab 26, 1067-1075.

10. Reyman M, Verrijn Stuart AA, van Summeren M et al. (2014) Vitamin D deficiency in childhood obesity is associated with high levels of circulating inflammatory mediators, and low insulin sensitivity. Int J Obes (Lond) 38, 46-52.

11. Atkinson MA, Melamed ML, Kumar J et al. (2014) Vitamin D, race, and risk for anemia in children. $J$ Pediatr 164, $153-158$.

12. Tang BM, Eslick GD, Nowson C et al. (2007) Use of calcium or calcium in combination with vitamin D supplementation to prevent fractures and bone loss in people aged 50 years and older: a meta-analysis. Lancet 370, 657-666. 
13. Avenell A, Cook JA, MacLennan GS et al. (2009) Vitamin D supplementation and type 2 diabetes: a sub-study of a randomized placebo-controlled trial in older people (RECORD trial, ISRCTN 51647438). Age Ageing 38, 606-609.

14. Baker MR, Peacock M \& Nordin BE (1980) The decline in vitamin D status with age. Age Ageing 9, 249-252.

15. MacLaughlin J \& Holick MF (1985) Aging decreases the capacity of human skin to produce vitamin $\mathrm{D}_{3}$.J Clin Invest 76, 1536-1538.

16. Ekwaru JP, Zwicker JD, Holick MF et al. (2014) The importance of body weight for the dose response relationship of oral vitamin D supplementation and serum 25-hydroxyvitamin D in healthy volunteers. PLoS One 9, e0111265.

17. Weishaar T \& Rajan S (2015) Importance of body weight and skin color in determining appropriate vitamin $\mathrm{D}_{3}$ supplement doses for children and adolescents. Pediatr Res 77, 370-375.

18. Dhaliwal R, Mikhail M, Feuerman M et al. (2014) The vitamin D dose response in obesity. Endocr Pract 20, 1258-1264.

19. Ng K, Scott JB, Drake BF et al. (2014) Dose response to vitamin D supplementation in African Americans: results of a 4-arm, randomized, placebo-controlled trial. Am J Clin Nutr 99, 587-598.

20. Centers for Diseases Control and Prevention, National Center for Health Statistics (2013) National Health and Nutrition Examination Survey data (database). http://www. cdc.gov/nchs/nhanes.htm (accessed October 2013).

21. Zipf G, Chiappa M, Porter KS et al. (2013) National health and nutrition examination survey: plan and operations, 1999-2010. Vital and Health Stat 1 issue 56, 1-37.

22. R Core Team (2014) R: a language and environment for statistical computing (version 3.1.1.). http://www.R-project. org/ (accessed March 2015).

23. Lumley T (2012) Survey analysis of complex survey samples (version 3.28-2). http://faculty.washington.edu/tlumley/ survey/ (accessed March 2015).

24. Lumley T (2004) Analysis of complex survey samples. J Stat Softw 9, 1-19.

25. Johnson CL, Paulose-Ram R \& Ogden CL (2013) National health and nutrition examination survey: analytic guidelines 1999-2010. Vital and Health Stat 2 issue 161, 1-24.

26. Farahati J, Nagarajah J, Gilman E et al. (2015) Ethnicity, clothing style, and body mass index are significant predictors of vitamin D insufficiency in Germany. Endocr Pract 21, 122-127.
27. Angurana SK, Angurana RS, Mahajan G et al. (2014) Prevalence of vitamin D deficiency in apparently healthy children in north India. J Pediatr Endocrinol Metab 27, $1151-1156$

28. Xiao JP, Zang J, Pei JJ et al. (2015) Low maternal vitamin D status during the second trimester of pregnancy: a crosssectional study in Wuxi, China. PLoS One 10, e0117748.

29. Keast DR, Wang X III \& Fulgoni VL (2007) Usual vitamin D intake in the United States by ethnic group: NHANES 2003-2004. FASEB J 21, 104.6.

30. Calvo MS, Whiting SJ \& Barton CN (2004) Vitamin D fortification in the United States and Canada: current status and data needs. Am J Clin Nutr 80, 6 Suppl., 1710S-1716S.

31. Cheng TY, Millen AE, Wactawski-Wende J et al. (2014) Vitamin D intake determines vitamin D status of postmenopausal women, particularly those with limited sun exposure. J Nutr 144, 681-689.

32. US Department of Health and Human Services (2014) The Surgeon General's Call to Action to Prevent Skin Cancer. Washington, DC: Office of the Surgeon General.

33. Kuskowska-Wolk A, Bergstrom R \& Bostrom G (1992) Relationship between questionnaire data and medical records of height, weight and body mass index. Int J Obes Relat Metab Disord 16, 1-9.

34. Looker AC, Dawson-Hughes B, Calvo MS et al. (2002) Serum 25-hydroxyvitamin D status of adolescents and adults in two seasonal subpopulations from NHANES III. Bone 305, 771-777.

35. Talib HJ, Ponnapakkam T, Gensure R et al. (2015) Treatment of vitamin D deficiency in predominantly Hispanic and Black adolescents: a randomized clinical trial. J Pediatr (Epublication ahead of print version).

36. Heaney RP (2011) Serum 25-hydroxyvitamin D is a reliable indicator of vitamin D status. Am J Clin Nutr 94, 619-620.

37. Berman LE, Fisher AL, Ostchega Y et al. (2001) Quality assurance (QA)/quality control (QC) processes for the National Health and Nutrition Examination Survey (NHANES). AMIA Annu Symp Proc 862. http://www.ncbi.nlm. nih.gov/pmc/articles/PMC2243447/pdf/procamiasymp000020901.pdf (accessed January 2016).

38. Yetley EA (2008) Assessing the vitamin D status of the US population. Am J Clin Nutr 88, issue 2, 558S-564S.

39. Holick MF, Binkley NC, Bischoff-Ferrari HA et al.; Endocrine Society (2011) Evaluation, treatment, and prevention of vitamin D deficiency: an Endocrine Society clinical practice guideline. J Clin Endocrinol Metab 96, 1911-1930. 